\title{
CMRF35-like molecule 1 (CLM-1) regulates eosinophil homeostasis by suppressing cellular chemotaxis
}

\author{
I Moshkovits $^{1}$, D Shik ${ }^{1}$, M Itan $^{1}$, D Karo-Atar ${ }^{1}$, B Bernshtein ${ }^{2}$, AY Hershko ${ }^{3}$, M van Lookeren Campagne ${ }^{4}$ \\ and A Munitz ${ }^{1}$
}

Eosinophil accumulation in health and disease is a hallmark characteristic of mucosal immunity and type 2 helper Tcell (Th2) inflammation. Eotaxin-induced CCR3 (chemokine (C-C motif) receptor 3) signaling has a critical role in eosinophil chemotactic responses. Nevertheless, the expressions of immunoreceptor tyrosine-based inhibitory motif-bearing receptors such as CMRF35-like molecule-1 (CLM-1) and their ability to govern eosinophil migration are largely unknown. We now report that CLM-1 (but not CLM-8) is highly and distinctly expressed by colonic and adipose tissue eosinophils. Furthermore, $\mathrm{Clm} 1^{-1-}$ mice display elevated baseline tissue eosinophilia. CLM-1 negatively regulated eotaxin-induced eosinophil responses including eosinophil chemotaxis, actin polymerization, calcium influx, and extracellular signal-regulated kinase (ERK)-1/2, but not p38 phosphorylation. Addition of CLM-1 ligand (e.g., phosphatidylserine) rendered wild-type eosinophils hypochemotactic in vitro and blockade of CLM-1/ligand interactions rendered wild-type eosinophils hyperchemotactic in vitro and in vivo in a model of allergic airway disease. Interestingly, suppression of cellular recruitment via CLM-1 was specific to eosinophils and eotaxin, as leukotriene $\mathrm{B}_{4}\left(\mathrm{LTB}_{4}\right)$ - and macrophage inflammatory protein-1 $\alpha$ (MIP-1 $\alpha$ )-induced eosinophil and neutrophil migration were not negatively regulated by CLM-1. Finally, peripheral blood eosinophils obtained from allergic rhinitis patients displayed elevated CLM-1/CD300f levels. These data highlight CLM-1 as a novel regulator of eosinophil homeostasis and demonstrate that eosinophil accumulation is constantly governed by CLM-1, which negatively regulates eotaxin-induced eosinophil responses.

\section{INTRODUCTION}

Eosinophils are bone marrow (BM)-derived cells that have key roles in homeostasis (e.g., metabolism and humoral immunity) and are implicated in the pathogenesis of numerous inflammatory processes. ${ }^{1}$ At baseline, eosinophils reside mainly in the gastrointestinal tract, which serves as the largest eosinophil reservoir. ${ }^{2}$ However, they are also found in substantial numbers in other organs, including the adipose tissue and uterus., Following type 2 helper $\mathrm{T}$ cell (Th2)-driven inflammatory responses or innate immune activation, such as those occurring in asthma and colitis, respectively, eosinophils are recruited into the inflamed tissue, where they modulate immune responses by releasing an array of molecules, including cytotoxic proteins, cytokines, and lipid mediators, and interact with various cell types. ${ }^{2,4}$
Eotaxin-1/CCL11, eotaxin-2/CCL24, and eotaxin-3/CCL26 encode for CC chemokines with eosinophil-selective chemoattractant activity. ${ }^{5-8}$ Together with their cognate receptor, CCR3 (chemokine (C-C motif) receptor 3), eotaxins play a fundamental role in eosinophil homing. ${ }^{9,10}$ In fact, eotaxins are critical for regulating baseline levels of eosinophils in the gastrointestinal tract and for the recruitment of eosinophils in settings of colonic inflammation and following allergen challenge in the lungs. ${ }^{1-17}$ Despite the key roles of eotaxins in eosinophil migration, additional non-eosinophil-specific chemotactic mediators, such as macrophage inflammatory protein 1 (MIP-1 $\alpha$ (CCL3)), regulated and normal $\mathrm{T}$ cell expressed and secreted (RANTES (CCL5)), and leukotrienes (LTs, especially $\mathrm{LTC}_{4}$ and $\mathrm{LTB}_{4}$ ), can promote eosinophil recruitment as well. ${ }^{18-20}$ Although the activation pathways

\footnotetext{
${ }^{1}$ Department of Clinical Microbiology and Immunology, Sackler School of Medicine, Tel-Aviv University, Tel-Aviv, Israel. ${ }^{2}$ Department of Immunology, Weizmann Institute of Science, Rehovot, Israel. ${ }^{3}$ Laboratory of Allergy and Clinical Immunology, Department of Medicine, The Herbert Center of Mast Cell Disorders, Meir Medical Center, Kfar Saba, Israel and ${ }^{4}$ Department of Immunology, Genentech, South San Francisco, California, USA. Correspondence: A Munitz (arielm@post.tau.ac.il)
} 
regulating eosinophil recruitment have been extensively studied, endogenous molecular pathways that are capable of restraining eosinophil accumulation have been scarcely examined. $^{21}$

The CMRF35-like molecule (CLM) family members consist of nine transmembrane glycoprotein receptors mapping to mouse chromosome 11D. Of these, CLM-1 and CLM-8 (human CD300f and CD300a, respectively) possess immunoreceptor tyrosine-based inhibitory motifs (ITIMs) in their intracellular domains and are thus able to suppress cellular activation by recruitment of phosphatases. ${ }^{22-24}$ The in vivo roles of CLM-1 have only recently been described, owing to the generation of $\mathrm{Clm}^{-1-}$ mice. These studies demonstrated key roles for CLM-1 in governing myeloid cell activities in experimental autoimmune encephalomyelitis and in mast cell-dependent allergic responses in mice. ${ }^{25,26}$ Despite these findings, the overall physiological function of CLM-1 is largely unknown and its expression and function in eosinophils has yet to be defined.

In this study, we demonstrate that CLM-1 is highly and uniquely expressed by adipose tissue and colonic eosinophils. Furthermore, we identify CLM-1 as a novel and specific negative regulator of eotaxin-induced responses in eosinophils including migration, calcium influx, actin polymerization, and intracellular signaling. To the best of our knowledge, this is the first reported evidence of a role for CLM-1 in the regulation of cellular migration. Our study highlights CLM-1 as a novel regulator of eosinophil homeostasis by specific negative regulation of cellular chemotaxis.

\section{RESULTS}

\section{Expression of CLM-1 and CLM-8 by murine eosinophils}

To define the expression of the ITIM-bearing CLM family members (e.g., CLM-1 and CLM-8) in murine eosinophils, we undertook a flow cytometric approach. Eosinophils (defined as $\mathrm{CD} 5^{+} / \mathrm{CCR}^{+}{ }^{+} /$Siglec-F $\mathrm{F}^{+} / \mathrm{SSC}^{\text {high }}$ cells; see Figure 3) from all the tested anatomical locations expressed CLM-1 and CLM-8 (Figure 1a and b). Interestingly, although peritoneal eosinophils displayed readily detectable levels of CLM-1, the expression of CLM-1 was nearly nondetectable in peritoneal macrophages (defined as $\mathrm{CD} 11 \mathrm{~b}^{+} / \mathrm{F} 480^{+}$cells; Figure 1b). This expression pattern was specific for CLM-1 as both peritoneal eosinophils and peritoneal macrophages expressed comparable levels of CLM-8 (Figure 1b). The finding that
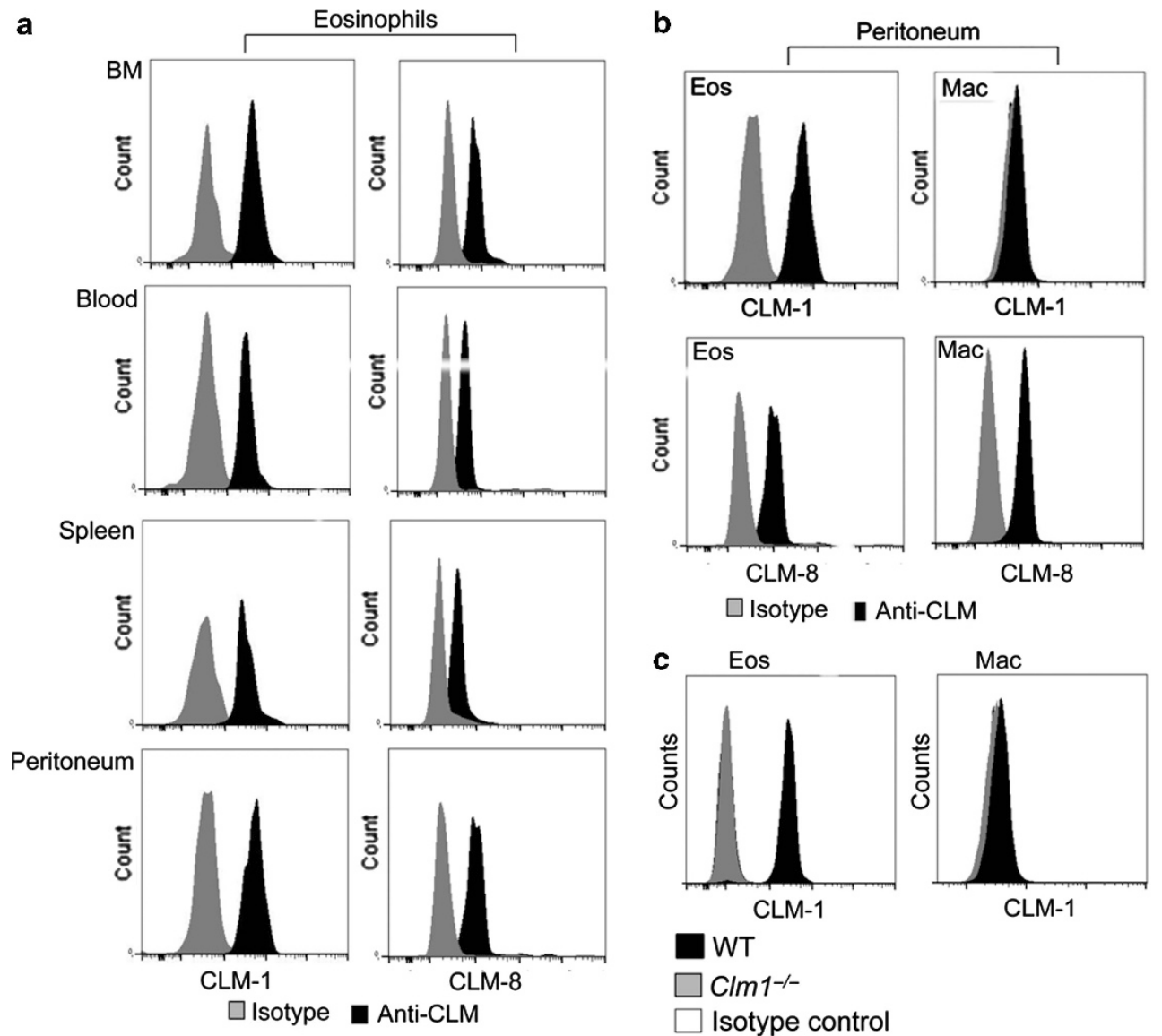

Figure 1 CLM-1 and CLM-8 are expressed by murine eosinophils. Single-cell suspensions from the indicated anatomical sources were obtained from wild-type (WT) or Clm1 $1^{-1-}$ mice. (a, b) Thereafter, the expression of CLM-1 and CLM-8 was examined on the surface of eosinophils and (b) macrophages. (c) Analysis of CLM-1 expression in peritoneal eosinophils and macrophages obtained from C/m $1^{-/-}$and WT mice. Representative histograms are shown from at least $n=8$. BM, bone marrow; CLM-1, CMRF35-like molecule-1; CLM-8, CMRF35-like molecule-8; Eos, eosinophil; Iso, isotype-matched control; Mac, macrophage; WT, wild-type. 
CLM-1 was not expressed under baseline conditions by peritoneal macrophages was further confirmed by staining $\mathrm{Clm} 1^{-/-}$peritoneal macrophages with anti-CLM-1 or isotype-matched control antibodies, which revealed no CLM-1 ${ }^{+}$macrophages (Figure 1c). Notably, anti-CLM-1stained $\mathrm{Clm}^{-/-}$cells displayed identical fluorescent intensity levels as the isotype control-stained $\mathrm{Clm} 1^{-/-}$cells.

\section{Unique expression of CLM-1 by adipose tissue and colonic eosinophils}

The unique expression of CLM-1 in peritoneal eosinophils in comparison with macrophages raised the possibility that tissue eosinophils would specifically express CLM-1 as well. Indeed, colonic and adipose tissue eosinophils (as defined by $\mathrm{CD} 45^{+} /$Siglec-F ${ }^{+} / \mathrm{CD} 11 \mathrm{~b}^{+}$cells) consistently expressed CLM-1 and CLM-8 (Figure 2a and b). Strikingly, however, CLM-1 expression was completely absent in colonic macrophages (defined by $\mathrm{CD}_{4} 5^{+} / \mathrm{MHC}-\mathrm{II}^{+} / \mathrm{CD} 11 \mathrm{~b}^{+} /$ CD11 $\mathrm{c}^{-}$cells), very low in dendritic cells (DCs, as defined by $\mathrm{CD} 5^{+} / \mathrm{MHC}^{-\mathrm{II}^{+}} / \mathrm{CD} 11 \mathrm{~b}^{-} / \mathrm{CD} 11 \mathrm{c}^{+}$cells, Figure $\mathbf{2 a}$ and $\left.\mathbf{d}\right)$ and nearly nondetectable in adipose tissue macrophages (defined as $\mathrm{CD} 45^{+} / \mathrm{CD} 11 \mathrm{~b}^{\text {high }} / \mathrm{F} 4 / 80^{+}$cells), respectively (Figure $\mathbf{2 b}$ and $\mathbf{d}$ ). Consistently, analysis of CLM-1 mRNA expression in sorted colonic macrophages and eosinophils revealed that eosinophils express markedly higher levels of CLM-1 mRNA levels in comparison with macrophages (Figure 2c). Interestingly, and in contrast to CLM-1 expression in adipose tissue and colonic macrophages, BM, splenic, and alveolar macrophages displayed readily detectable levels of CLM-1 (see Supplementary Figure S1 online).

Analysis of CLM-1 levels in peripheral blood neutrophils and monocytes $\left(\mathrm{Ly}_{6 \mathrm{G}}{ }^{+} / \mathrm{CD} 11 \mathrm{~b}^{+}\right.$and $\mathrm{Ly} 6 \mathrm{C}^{\text {high }} / \mathrm{CD} 11 \mathrm{~b}^{+}$cells, respectively) revealed that circulating neutrophils and monocytes express similar levels of CLM-1 as peripheral blood eosinophils (Figure 2e; also see Figure 1a). Collectively, these results demonstrate that under homeostatic conditions, the highest CLM-1-expressing cells in the colon and adipose tissue are eosinophils, at least in comparison with macrophages and DCs.

\section{CLM-1 regulates baseline tissue eosinophil levels}

The unique expression for CLM-1 in eosinophils prompted us to assess eosinophil levels in $\mathrm{Clm1}^{-/-}$mice. BM and peripheral blood eosinophil levels were comparable between wild-type (WT), $C \operatorname{lm} 1^{-/-}$, and $C \operatorname{lm} 8^{-/-}$mice (Figure 3a, b and $\mathbf{g}$ ). In contrast, $\mathrm{Clm} 1^{-1-}$ mice displayed a two- to three-fold increase in peritoneal, gastrointestinal, and adipose tissue eosinophil levels as compared with WT and $\mathrm{Clm} 8^{-/-}$mice (Figure 3c-e and g). Anti-eosinophil major basic protein (MBP) staining revealed increased $\mathrm{MBP}^{+}$cells in the colonic lamina propria of $\mathrm{Clm}^{-/-}$mice. Interestingly, the colon of $\mathrm{Clm}^{-1-}$ mice displayed sporadic areas with eosinophilic lesions (Figure 3f, upper right panel). No alterations were observed in macrophage and/or other cellular components in the $\mathrm{Clm}^{-/-}$mice (data not shown).

Importantly, increased baseline eosinophilia in $\mathrm{Clm}^{-/-}$ mice was not due to overproduction of eotaxins or alteration in CCR3 receptor levels, as despite elevated tissue eosinophilia, $\mathrm{Clm}^{-/-}$mice displayed decreased baseline eotaxin-1 and eotaxin-2 levels in the colon and had no difference in eotaxin-1 and/or eotaxin-2 expression in the peritoneal cavity and/or adipose tissue. Moreover, $\mathrm{Clm}^{-/-}$eosinophils expressed similar CCR3 surface levels when compared with WT eosinophils (Supplementary Figure S2 online).

\section{CLM-1 negatively regulates eotaxin-induced eosinophil responses}

As BM and blood eosinophil levels were similar in WT and $\mathrm{Clm} 1^{-1-}$ mice (Figure 3a and $\mathbf{b}$ ), yet $\mathrm{Clm} 1^{-1-}$ mice displayed elevated tissue eosinophilia (Figure $\mathbf{3 c - e}$ ), we hypothesized that CLM-1 negatively regulates eosinophil trafficking. To examine this hypothesis, in vitro chemotaxis assays were performed. Indeed, following stimulation with eotaxin-1 or eotaxin-2, Clm1 $1^{-/-}$low-density BM-derived eosinophils displayed significantly increased chemotaxis in a dose-dependent manner (Figure $\mathbf{4 a}$ and $\mathbf{b}$ ). For example, addition of $100 \mathrm{ng} \mathrm{ml}^{-1}$ eotaxin-1 or eotaxin-2 to the lower chambers of the trans-well system induced a 10-fold increase in WT eosinophil chemotaxis, whereas $\mathrm{Clm1}^{-1-}$ eosinophils displayed a 20- to 25-fold increase (Figure 4a, b). Consistently, eotaxin-1-induced calcium influx was increased in $\mathrm{Clml}^{-/-}$ eosinophils in comparison with WT eosinophils (Figure 4c). Furthermore, $\mathrm{Clm1}^{-/-}$eosinophils displayed enhanced and sustained actin polymerization (Figure 4d). Of note, similar results were obtained with primary peritoneal eosinophils (data not shown).

Assessment of eotaxin-1-induced signaling in $\mathrm{Clm}^{-/-}$ eosinophils revealed that CLM-1 specifically regulated eotaxin1-induced extracellular signal-regulated kinase (ERK)-1/2 phosphorylation but not that of p38 (Figure 4e and f). Indeed, eotaxin-1-treated WT eosinophils displayed a $30 \pm 5 \%$ increase in ERK phosphorylation as early as $1 \mathrm{~min}$ after eotaxin1 exposure, whereas at this same time point $\mathrm{Clm}^{-1-}$-treated cells displayed a $95.2 \pm 12 \%$ increase (Figure 4e). Furthermore, even after $5 \mathrm{~min}$ of eotaxin-1 stimulation, when ERK phosphorylation in WT cells decreased below baseline levels $(-18.3 \pm 2.4 \%)$, ERK phosphorylation was still significantly higher than baseline in $\mathrm{Clm}^{-1-}$ eosinophils $(45.3 \pm 16.2 \%$;

Figure 2 CMRF35-like molecule-1 (CLM-1) is highly expressed by tissue-resident eosinophils. (a, b) CLM-1 and CLM-8 expression was assessed in colonic and adipose tissue cells (histogram plots) and (d, e) quantitative analysis of $\Delta$ median fluorescence intensity ( $\Delta \mathrm{MFI}$ ) was calculated. (c) Quantitative PCR analysis of CLM-1 mRNA expression in resident colonic eosinophils and macrophages is shown. Clm1 levels were assessed and normalized to the house keeping gene hypoxanthine-guanine phosphoribosyltransferase (Hprt). (e) Analysis of CLM-1 expression in peripheral blood neutrophils and monocytes is shown. Representative histograms are shown from at least $n=5$. DC, dendritic cell; Eos, eosinophil; FSC, forward scatter; Mac, macrophage; MHC II, major histocompatibility complex class II; Mono, monocyte; Neut, neutrophil; NS, nonsignificant; Siglec-F, Sialic acid-binding immunoglobulin-like lectin-F; SSC, side scatter. ${ }^{\star} P<0.05$ and ${ }^{* \star \star} P<0.001$. 

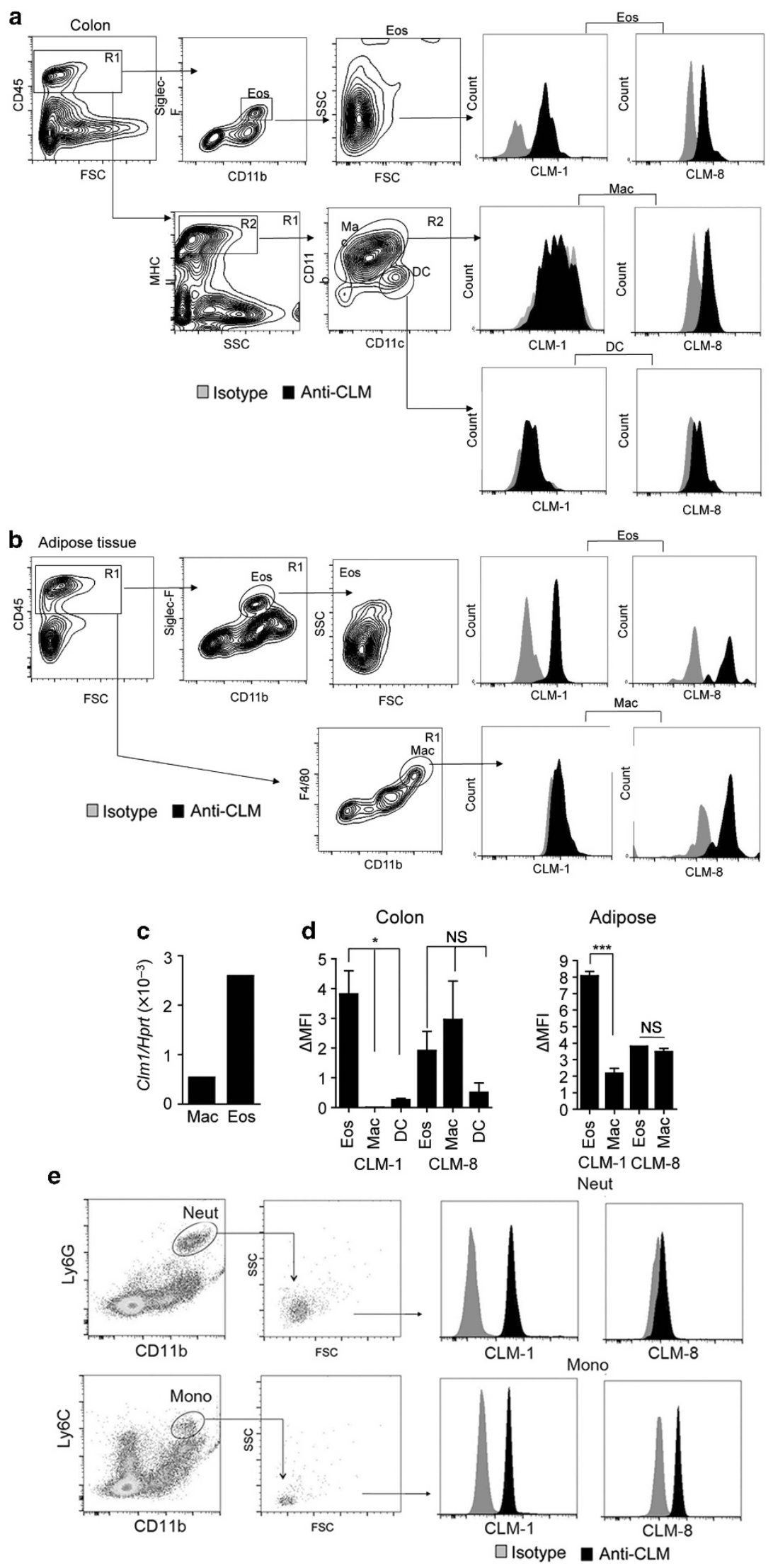
Figure 4e). Assessment of chemokine-induced adhesion molecule upregulation revealed that eotaxin-1-induced CD11b levels were similar in WT and $\mathrm{Clm}^{-/-}$ eosinophils (Supplementary Figure S3 online).

To further establish the role of CLM-1 in eotaxin-1-induced eosinophil chemotaxis, a direct in vivo approach was used in which eotaxin-1 was directly injected into the peritoneal cavity and eosinophil recruitment was assessed. Consistent with previous publications, ${ }^{27}$ administration of eotaxin alone was not sufficient to induce significant eosinophil accumulation in the peritoneal cavity of WT mice (Figure $\mathbf{4 g - i}$ ). In contrast, eotaxin-1 administration to $\mathrm{Clm}^{-/-}$mice induced a significant influx of eosinophils to the peritoneal cavity, with their levels reaching $17-24 \%$ of all peritoneal leukocytes (Figure 4g-i).

\section{CLM-1 does not regulate MIP-1 $\alpha$ - and LTB $_{4}$-induced eosinophil or neutrophil chemotaxis}

Next, we determined whether the inhibitory function of CLM-1 is specific to CCR3 ligands, or if it can suppress additional chemotactic signals in eosinophils. In contrast to our findings with eotaxin-1 and eotaxin-2 (Figure $\mathbf{4 a}$ and $\mathbf{b}$ ), the chemotactic responses of $C l m 1^{-/-}$and WT eosinophils to MIP- $1 \alpha$ and $\mathrm{LTB}_{4}$ were similar (Figure 5a and b). Supporting these results, ex vivo neutrophil chemotaxis assays using naive splenocyte cell suspension demonstrated that $\mathrm{Clm}^{-/-}$ a
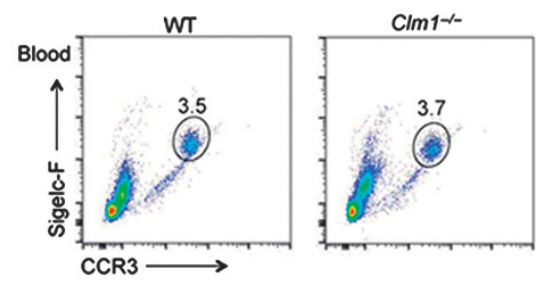

C

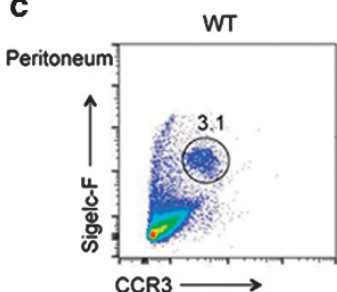

e
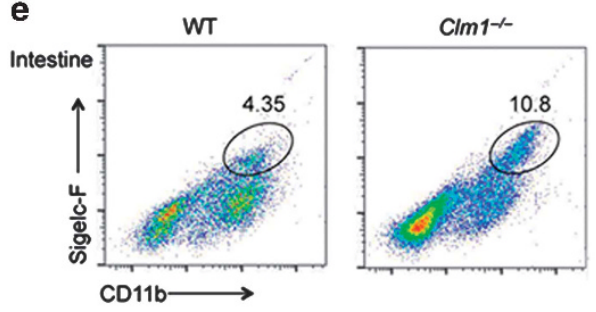

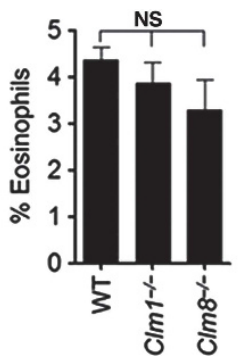

b
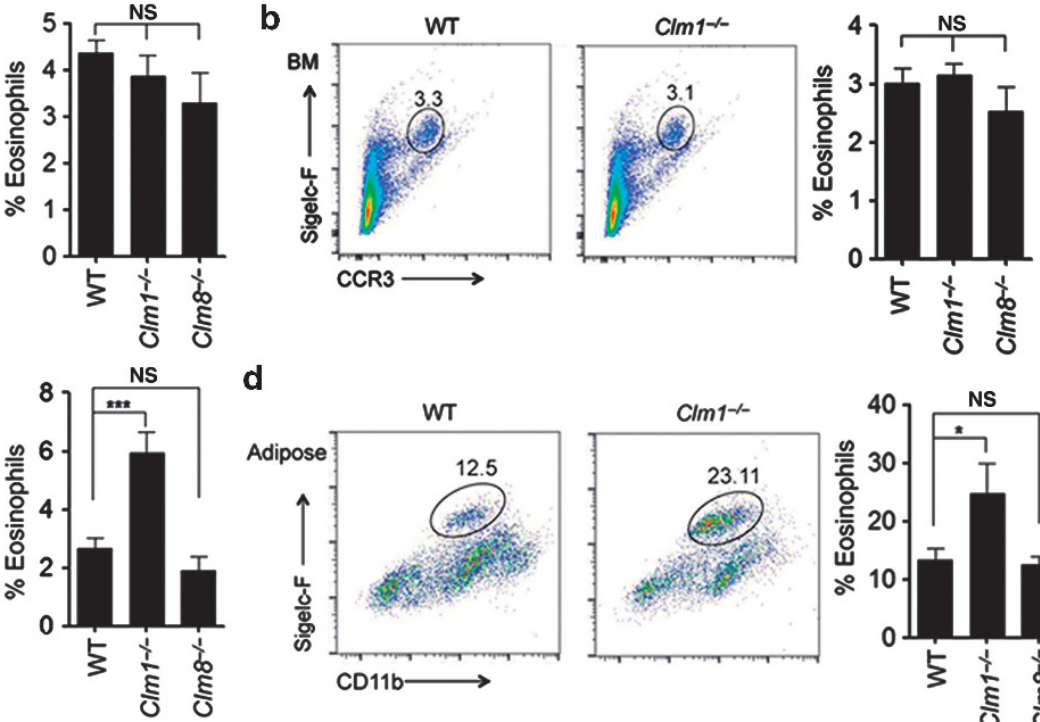

d
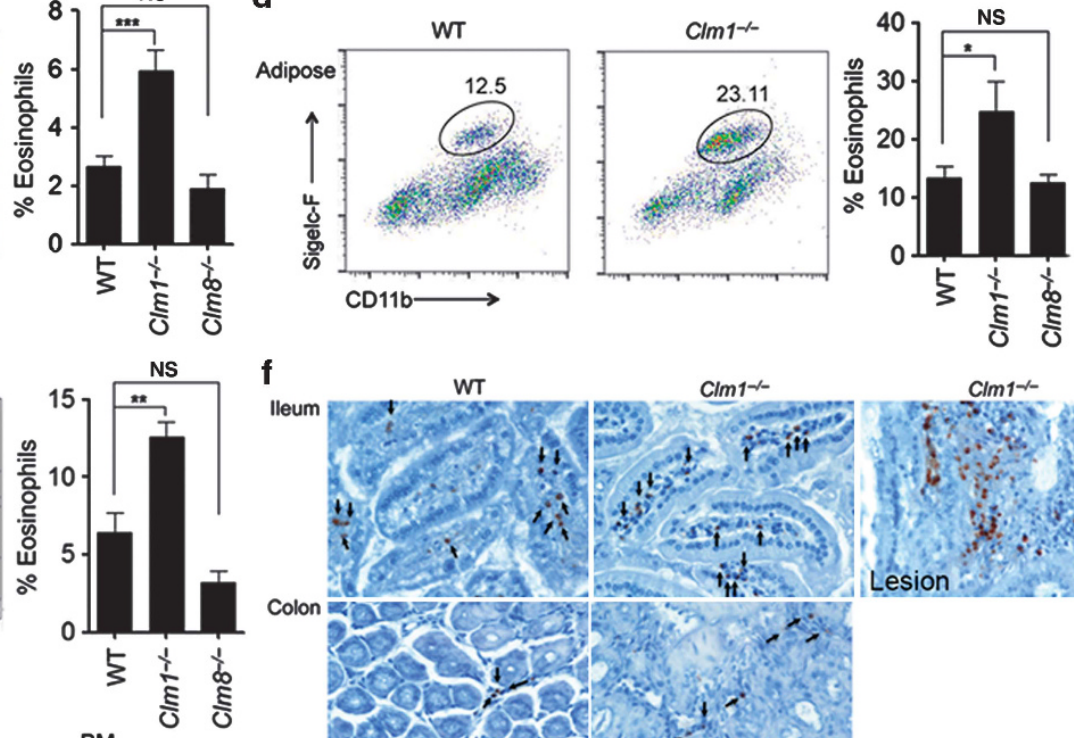

f
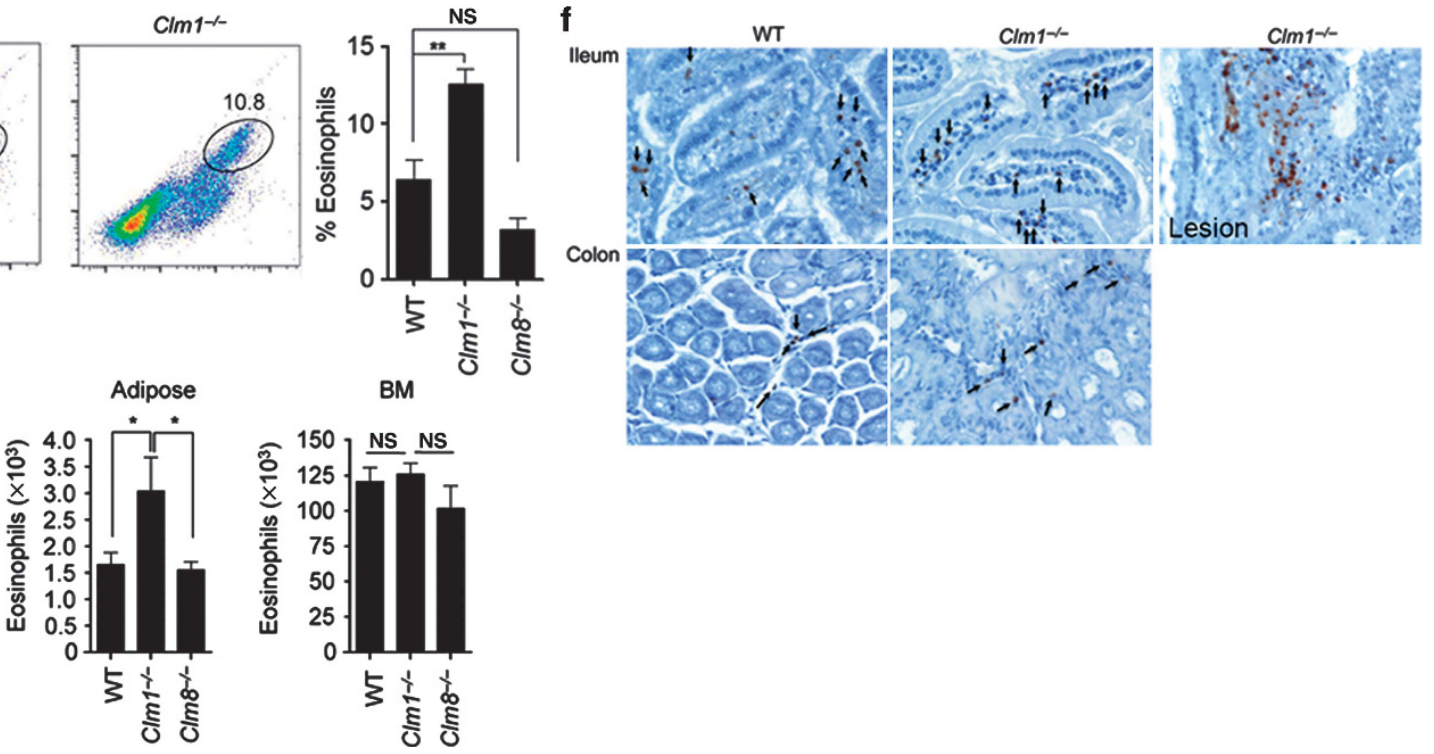

Figure 3 Increased baseline eosinophilia in $\mathrm{Clm1^{-/- }}$ but not $\mathrm{Clm} 8^{-/-}$mice. (a-e) Density plots demonstrating eosinophil percentages in wild-type (WT) and $C / \mathrm{m}^{-/-}$mice. Quantitative analysis of eosinophil percentages in the different organs of WT, $C / m 1^{-/-}$, and $C / m 8^{-/-}$mouse is shown as were assessed by flow cytometry. (f) A representative photomicrograph of anti-eosinophil major basic protein (MBP) immunohistochemistry is shown (original magnification $\times 40$ ) in the ileum (upper photomicrographs) and colon (lower photomicrographs) of WT and Clm $1^{-/-}$mice (left and right photomicrographs, respectively). In addition, a representative eosinophilic lesion in the colon of $\mathrm{Clm} 1^{-/-} \mathrm{mice}$ is shown; black arrows indicate $\mathrm{MBP}+$ cells representing eosinophils. (g) Quantitative analysis of total eosinophil counts in the colon, adipose tissue, and bone marrow (BM) of WT, Clm1-/- , and $\mathrm{Clm} 8^{-/-}$mice. Representative density plots are shown from at least $n=6$. CLM, CMRF35-like molecule; NS, nonsignificant; Siglec-F, Sialic acid-binding immunoglobulin-like lectin-F. ${ }^{\star} P<0.05,{ }^{\star *} P<0.01$, and ${ }^{\star * \star} P<0.001$. 

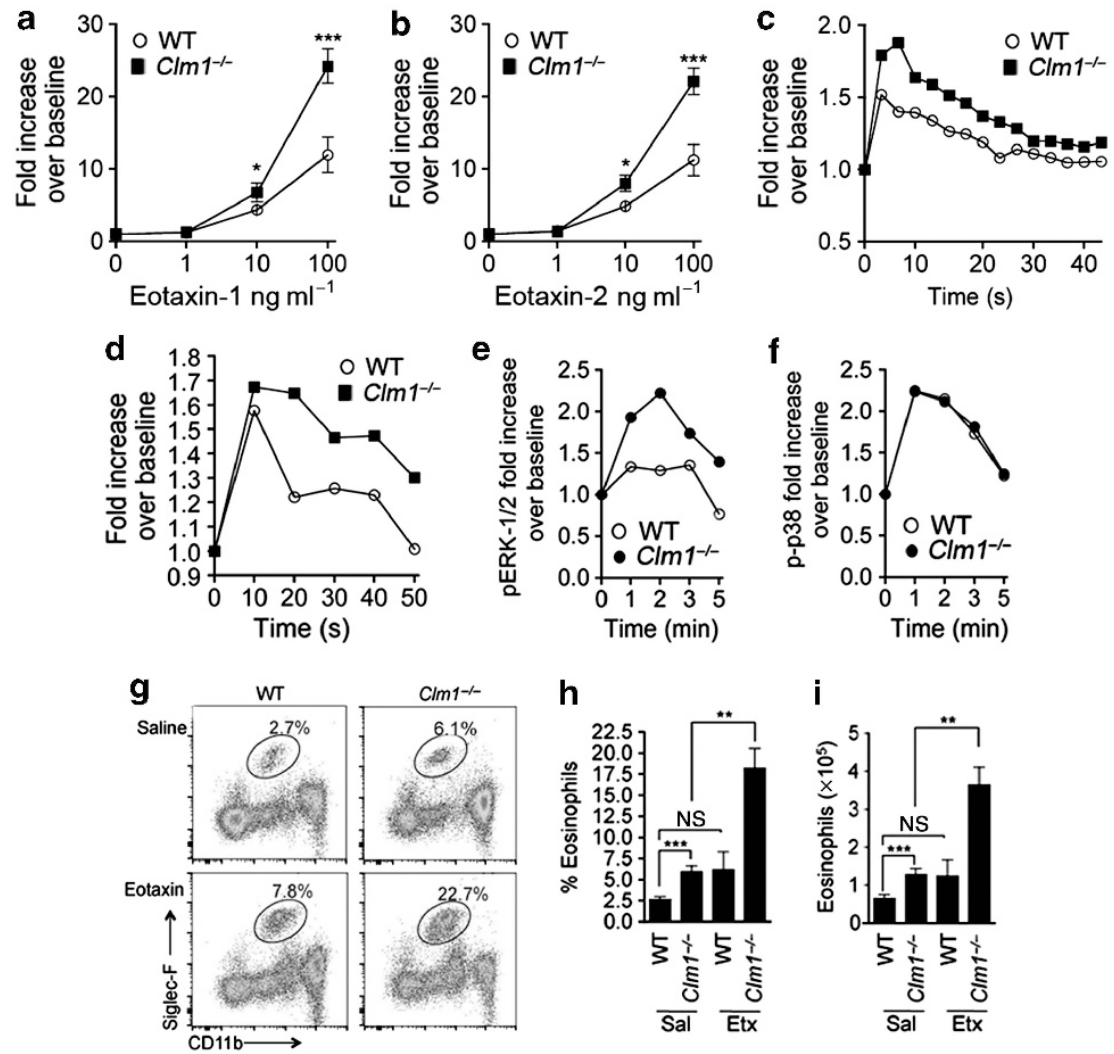

Figure 4 CMRF35-like molecule-1 (CLM-1) is a negative regulator of eotaxin-induced eosinophil responses. Wild-type (WT) and Clm1 ${ }^{-/-}$low-density bone marrow (LDBM)-derived eosinophils were subjected to in vitro (a, b) chemotaxis, (c) calcium influx, (d) actin polymerization, and (e) extracellular signalregulated kinase-1/2 (ERK-1/2) and (f) p38 phosphorylation assays. (g-i) WT and C/m1 ${ }^{-/-}$mice were injected with eotaxin-1 (Etx) intraperitoneally and eosinophil transmigration into the peritoneal cavity was assessed. (a-f) Representative results and (g) density plots are shown from at least $n=6$ experiments. NS, nonsignificant; Sal, saline; Siglec-F, Sialic acid-binding immunoglobulin-like lectin-F. ${ }^{\star} P<0.05$, ${ }^{* \star} P<0.01$, and ${ }^{* \star \star} P<0.001$.

a

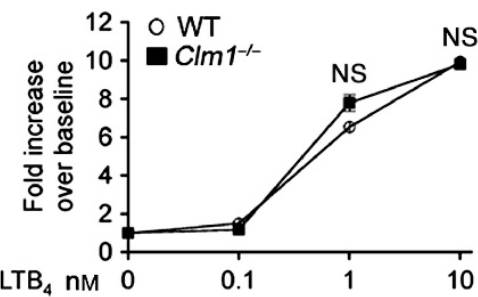

c

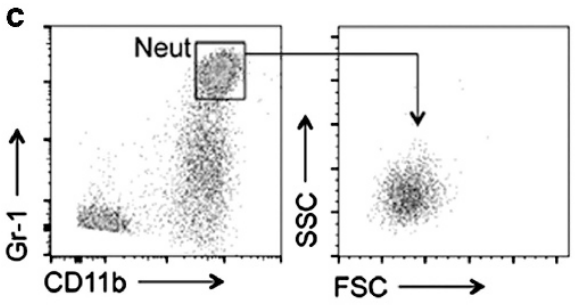

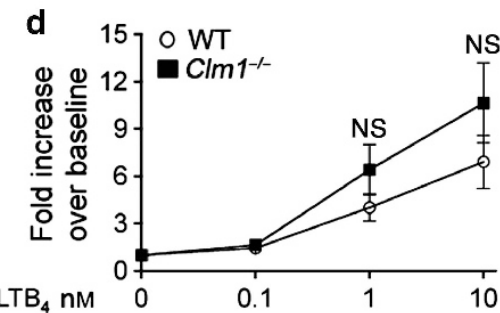
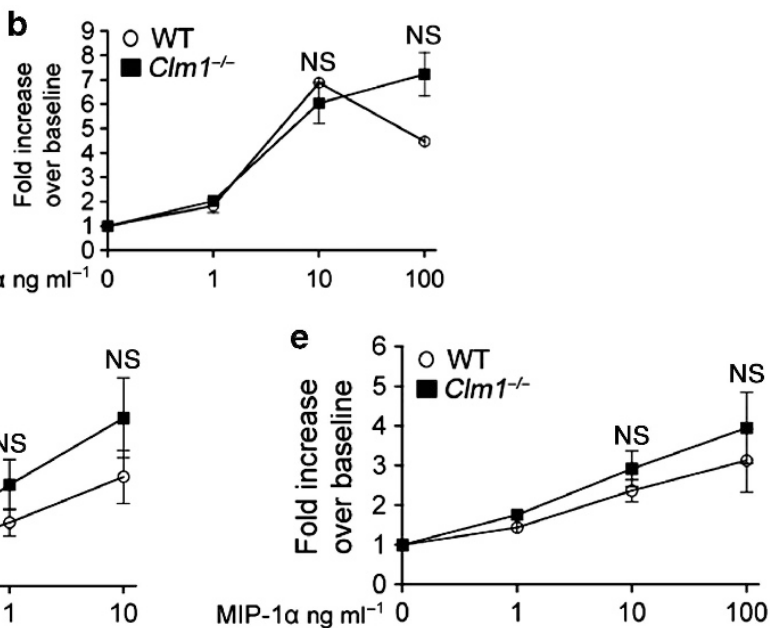

Figure 5 CMRF35-like molecule-1 (CLM-1) does not regulate eosinophil or neutrophil chemotactic responses toward leukotriene $B_{4}\left(L_{T} B_{4}\right)$ and macrophage inflammatory protein-1 $\alpha$ (MIP-1 $\alpha)$. (a, b) Eosinophils or (d, e) splenocytes were obtained from wild-type (WT) and Clm1 $1^{-/-}$mice and subjected to chemotaxis assays using (a, d) LTB ${ }_{4}$ and (b, e) MIP-1 $\alpha$ at the indicated concentrations. (c) The gating strategy for detection of splenic neutrophils is depicted; $n=3$. FSC, forward scatter; Neut, neutrophil; NS, nonsignificant; SSC, side scatter.

neutrophils (as determined by $\mathrm{CD}_{4} 5^{+} / \mathrm{CD} 11 \mathrm{~b}^{+} / \mathrm{Gr}-1^{+} /$ $\mathrm{SSC}^{\text {int }}$ ) displayed essentially the same chemotactic activity as WT neutrophils in response to $\mathrm{LTB}_{4}$ and MIP- $1 \alpha$ stimulation (Figure 5c-e). Thus, CLM-1 is a novel regulator of eotaxininduced eosinophil chemotaxis.
CLM-1 binds apoptotic eosinophils and regulates eosinophil chemotaxis via receptor/ligand interactions

The finding that $\mathrm{Clm}^{-/-}$eosinophils displayed increased responses to eotaxin-induced stimulation in vitro suggested that CLM-1/ligand interactions are required to suppress 

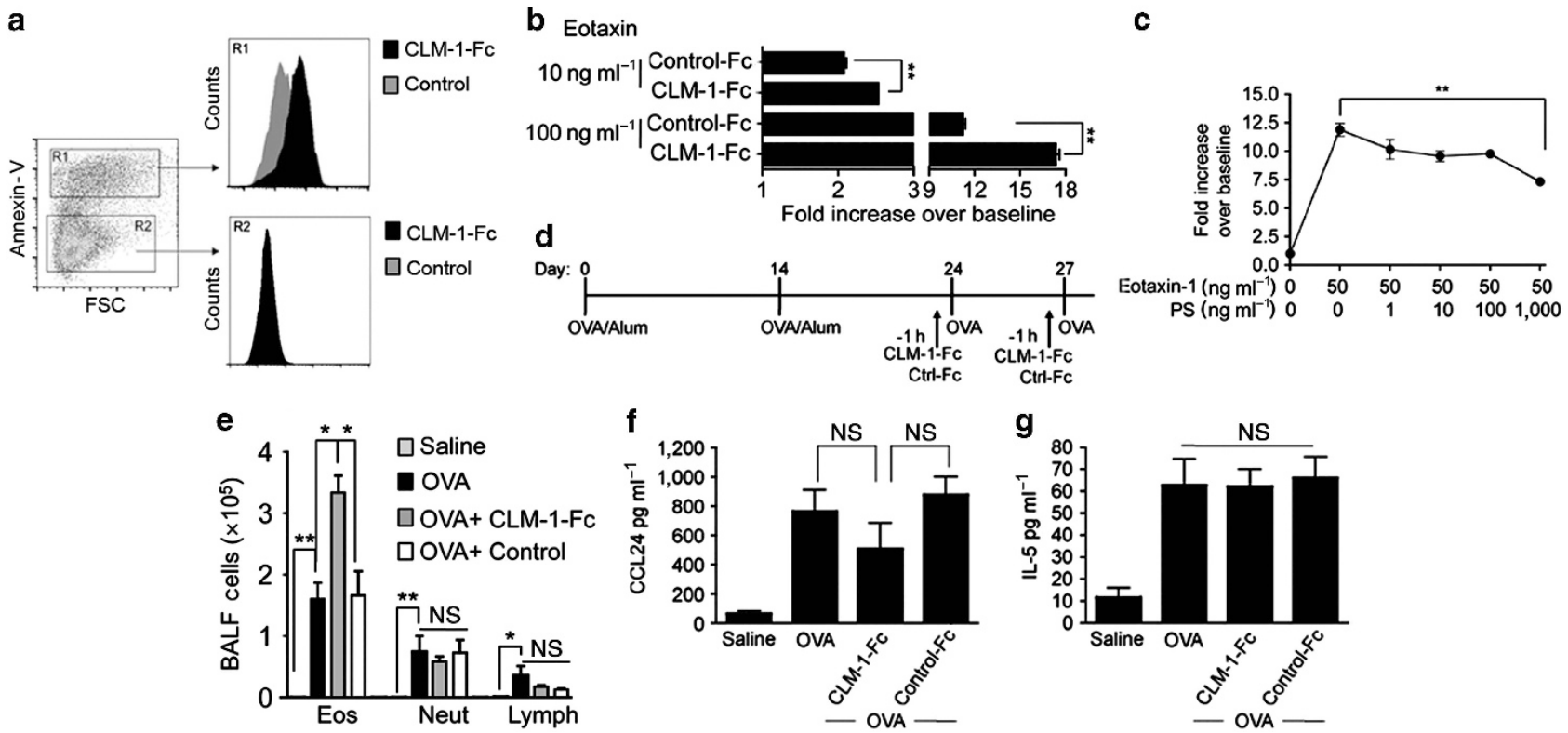

Figure 6 CLM-1/ligand interactions regulate eosinophil accumulation during allergic airway inflammation. (a) Low-density bone marrow (LDBM)derived eosinophils were stained with Annexin-V-FITC and CLM-1-Fc or control-Fc fusion protein and the binding of CLM-1 to apoptotic eosinophils was assessed by flow cytometry. LDBM-derived eosinophils were treated with (b) CLM-1-Fc, control-Fc fusion proteins, or (c) phosphatidylserine (PS) and subjected to in vitro chemotaxis assays in response to eotaxin-1. (d) Wild-type (WT) mice were sensitized with OVA/Alum and $1 \mathrm{~h}$ before allergen- challenge were intranasally treated with CLM-1-Fc or control-Fc fusion protein; at $24 \mathrm{~h}$ after the last OVA challenge, the mice were killed and bronchoalveolar lavage (BAL) was obtained for (e) differential cell counts, (f) CCL24 and (g) IL-5 expression; $n=6$. CLM-1, CMRF35-like molecule-1; Eos, eosinophil; FITC, fluorescein isothiocyanate; FSC, forward scatter; IL-5, interleukin 5; Lymph, lymphocyte; Neut, neutrophil; NS, nonsignificant; OVA, ovalbumin. ${ }^{\star} P<0.05$ and ${ }^{* *} P<0.01$.

eotaxin-induced eosinophil chemotaxis. CLM-1 was recently demonstrated to bind phosphatidylserine, which is expressed on the surface of apoptotic cells. ${ }^{28}$ Thus, we assessed the possibility that CLM-1 binds apoptotic eosinophils. To this end, BM-derived eosinophils were stained with Annexin- $\mathrm{V}$ together with IgG1 CLM-1-Fc or control fusion proteins. CLM-1 was capable of binding apoptotic eosinophils (as determined by annexin- $\mathrm{V}^{+}$cells) but not viable cells (Figure 6a). To further explore the role of CLM-1/ligand interactions in the regulation of eosinophil chemotaxis, WT eosinophils were incubated in the presence of IgG1 CLM-1-Fc fusion protein, which competes with cellular-expressed CLM-1 for ligand binding, resulting in decreased CLM-1 signaling. ${ }^{26}$ Thereafter, the cells were stimulated with eotaxin and assessed for their chemotactic activity. Notably, CLM-1-Fc fusion protein-treated WT eosinophils displayed significantly increased chemotaxis in response to eotaxin in comparison with that of control Fcfusion protein-treated cells (Figure $\mathbf{6 b}$ ). Furthermore, addition of increasing concentrations of phosphatidylserine to WT eosinophil chemotaxis assays revealed a significant reduction in the ability of eosinophils to respond to eotaxin (Figure 6c). Collectively, these data demonstrate a key role for CLM-1/ ligand interactions in the regulation of eosinophil chemotaxis

\section{CLM-1/ligand interactions regulate eosinophil accumulation during allergic airway inflammation}

Next, we were interested to determine whether eosinophil migration will be regulated by CLM-1 in the context of eosinophilic airway disease, which induces an eotaxin-dependent chemotactic gradient accountable for eosinophil accumulation. ${ }^{29}$ As CLM-1 is expressed by various cells including DCs and B cells, ${ }^{24}$ which are required for the sensitization phase of allergic airway inflammation, we chose a model that would assess the function of CLM-1 in the effector phase of allergic airway disease without affecting sensitization (Figure 6d). To this end, WT mice were sensitized twice with ovalbumin (OVA)/Alum (on days 0 and 14). Thereafter, $1 \mathrm{~h}$ before the subsequent OVA challenges, the mice were intranasally administered a CLM-1-Fc (or control-Fc) fusion protein (Figure 6d).

Assessment of eosinophil accumulation in the lungs following the last OVA challenge revealed a specific and significant increase in eosinophil accumulation in the CLM-1$\mathrm{Fc}$ fusion protein-treated mice in comparison with control-Fc fusion-treated mice (Figure 6e). Increased eosinophil accumulation was not due to increased eosinophil survival factors (e.g., interleukin 5 (IL-5)) or chemokines (e.g., eotaxins) as the levels of these mediators in the bronchoalveolar lavage fluid were similar in the mice treated with OVA, OVA + Control-Fc, and OVA + CLM-1-Fc fusion proteins (Figure 6f, g).

\section{Human peripheral blood eosinophils express CD300f that is} upregulated in active allergic rhinitis patients

Given the expression of CLM-1 by murine eosinophils, we next aimed to identify whether human eosinophils express CD300f, the human ortholog of CLM- $1 .{ }^{24}$ Although CD300f is known to be expressed and functional in various human myeloid cells, its 
a
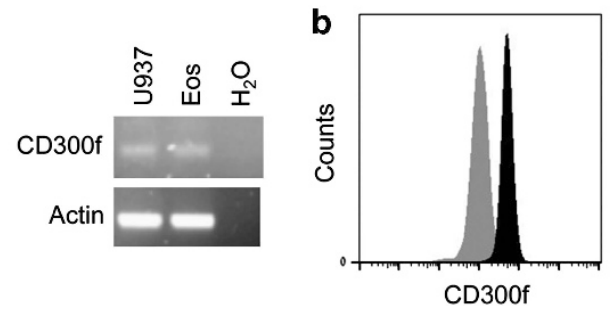

c

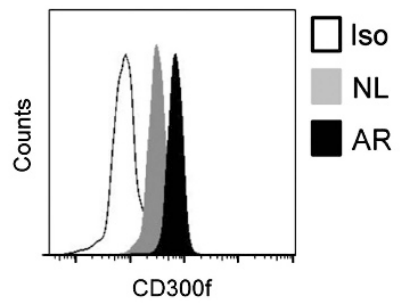

d

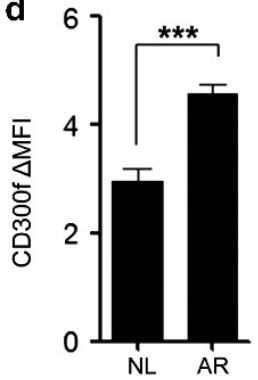

Figure 7 CD300f is expressed by human peripheral blood eosinophils and is upregulated on the surface of cells from allergic rhinitis patients. (a) The expression of CD300f was assessed by PCR in the U937 monocytic cell line (left lane), peripheral blood eosinophils (middle lane), or $\mathrm{H}_{2} \mathrm{O}$ control (right lane) and by (b) flow cytometry in normal peripheral blood eosinophils. In addition, a representative (c) histogram plot and (d) quantitative analysis of CD300f expression as assessed in eosinophils from healthy individuals (NL, $n=8)$ and allergic rhinitis (AR, $n=8)$ patients are shown. (a) Gel photograph and (b) histogram are representative of at least $n=3$. Eos, eosinophil; Iso, isotype-matched control; $\Delta \mathrm{MFI}, \Delta$ median fluorescence intensity. ${ }^{\star * *} P<0.001$.

expression and function in human eosinophils is unknown. To this end, eosinophils were purified from the peripheral blood of healthy volunteers and CD300f expression was examined by PCR (Figure 7a). Human eosinophils expressed substantial levels of CD300f, similar to those expressed by the monocytic cell line U937 (Figure 7a). Flow cytometric analysis confirmed that eosinophils express readily detectable levels of CD300f on their surface (Figure $7 \mathbf{b}$ ).

Previous studies have shown that diverse stimuli can induce a rapid upregulation of inhibitory receptors that then act as a negative feedback loop to restrain cellular activation. ${ }^{30}$ Flow cytometric analysis of CD300f expression by peripheral blood eosinophils in allergic rhinitis patients (as defined by $\mathrm{CD} 45^{+}$/ Siglec- $8^{+} / \mathrm{CCR} 3^{+} / \mathrm{CD} 16^{-} / \mathrm{CD} 14^{-} / \mathrm{SSC}^{\text {high }}$ ) revealed significantly elevated levels of CD300f surface expression in comparison with healthy individuals (Figure $7 \mathbf{c}$ and $\mathbf{d}$ ).

\section{DISCUSSION}

The migration of inflammatory cells from the blood stream into interstitial spaces is a cornerstone of the inflammatory process and critically regulated by chemokines. Specifically, eotaxins, via their respective CCR3 receptor, play a fundamental role in governing eosinophil migration in health and disease. ${ }^{1,2,14,31}$ Therefore, endogenous molecular pathways that can counterregulate the migratory responses of eosinophils may have considerable implications for disease therapy as specific inhibitory receptor agonists could be designed to curtail eosinophil accumulation. ${ }^{32-34}$ In an attempt to define novel negative regulators of eosinophil functions, we examined the

expression and function of CLM-1 in eosinophils. We demonstrated that: (i) CLM-1 is highly and almost exclusively expressed by colonic and adipose tissue eosinophils in comparison with other resident myeloid cells; (ii) the selective expression of CLM-1 in eosinophils corresponds with increased baseline eosinophilia in $\mathrm{Clm} 1^{-/-}$but not $\mathrm{Clm} 8^{-1-}$ mice; (iii) CLM-1 is a specific regulator of eotaxin-induced eosinophil responses in vitro (e.g., chemotaxis, calcium influx, actin polymerization, and signaling) and in vivo; (iv) CLM-1/ ligand interactions govern eosinophil migration in vitro and in settings of allergic airway disease; and finally (v) human eosinophils express CD300f that is upregulated in peripheral blood eosinophils obtained from allergic rhinitis patients. Collectively, our results demonstrate a novel physiological role for CLM-1 in eosinophil homeostasis by governing eotaxininduced eosinophil responses. These data substantially enhance our understanding of the molecular mechanisms that govern eosinophil accumulation in the tissue in health and disease.

Although eosinophils have been a therapeutic target for many years, there is a surprising paucity of studies regarding the expression and function of ITIM-bearing receptors that are expressed by these cells, especially in vivo. The CLM family of receptors consists of nine members; of these, CLM-1 and 8 (human CD300f and CD300a, respectively) are considered inhibitory as they contain various ITIMs in their intracellular domain and can recruit cellular phosphatases consequently suppressing cellular activation. ${ }^{24}$ Indeed, our findings highlight an exclusive role for CLM-1 in the negative regulation of eosinophils. Recent studies (including our own) emphasize the importance of ITIM-bearing receptors in eosinophil homeostasis via regulation of various signaling pathways. For example, mice that lack most of the cytoplasmic domain of the ITIM-bearing receptor, signal regulatory protein (SIRP)- $1 \alpha$ (SIRP $\alpha$ Cyto $^{-1-}$ mice), or CD 47 , the ligand for SIRP- $1 \alpha$, display elevated degranulation of intestinal eosinophils. ${ }^{35}$ Interestingly, this degranulation was associated with fewer lamina propria eosinophils, likely due to regulation of IL-5-induced eosinophil survival by SIRP- $1 \alpha{ }^{35}$ Furthermore, gastrointestinal eosinophils express the pan-B-cell marker CD22, and although the mechanism for this is unclear, $C d 22^{-/-}$mice display elevated gastrointestinal eosinophilia. In addition, we have recently shown that paired immunoglobulin-like receptor B (PIR-B) has a complex role in the regulation of eosinophil migration. Although PIR-B suppressed eosinophil migration in response to eotaxins, it positively regulated $\mathrm{LTB}_{4}$-induced migratory responses. The ability of PIR-B to suppress cellular migration was not confined to eosinophils as it could negatively regulate DCs and neutrophil chemotaxis as well. ${ }^{36,37}$ Of note, PIR-B was capable of suppressing MIP- $1 \alpha$-induced neutrophil chemotaxis. Thus, to the best of our knowledge, this is the first report demonstrating an endogenous receptor-mediated pathway (i.e., CLM-1) that can specifically suppress eotaxin-induced responses in eosinophils.

Mechanistically, we have shown that CLM-1 negatively regulates eotaxin-induced calcium influx, actin polymerization, 
and phosphorylation of ERK but not p38. It is important to note that although activation of eosinophils with CCR3 ligands has been shown to induce calcium influx and cytoskeleton rearrangements, suppression of eosinophil transmigration was not always associated with alterations in calcium influx. Most relevant, we have recently shown that induction of an inhibitory signaling cascade in human eosinophils by CD300a using artificial antibody crosslinking resulted in suppression of eosinophil chemotaxis in vitro. ${ }^{38}$ Interestingly and despite decreased chemotaxis in CD300a-activated cells, eotaxininduced calcium influx was not altered. ${ }^{38}$ Similarly, Fulkerson et al. ${ }^{39}$ have shown that inhibition of eosinophil transmigration by Mig does not require the inhibition of calcium influx. It is likely that the ability of ITIM-bearing receptors such as CD300a or CLM-1 to suppress calcium influx is dependent on their interaction with downstream adaptor molecules that are recruited to the receptor following cellular activation. Alternatively, it has recently been shown that all CD300 family members interact with each other, even with themselves and are capable of forming both homo- and heterodimers. Thus, the inhibitory signaling capacity of each CD300/CLM family member may be dependent on the expression of additional CD300/CLM-molecules. ${ }^{40}$ Defining the full expression and functional spectrum of CD300/CLM-1 family members in eosinophils will likely shed more light into these interactions and their relevance in eosinophil biology.

Importantly, the increased responsiveness of $\mathrm{Clm}^{-1-}$ eosinophils to eotaxin was not due to elevated levels of CCR3 on the surface of $\mathrm{Clm}^{-/-}$eosinophils or to increased tissue eotaxin levels. In fact, the expression of eotaxin was markedly reduced in the colon of $\mathrm{Clm}^{-1-}$ mice. This observation may be partially explained by the fact that $C \operatorname{lm} 1^{-/-}$eosinophils have increased eotaxin consumption. Alternatively, it is possible that albeit low expression levels, CLM-1 regulates baseline eotaxin production by resident colonic myeloid cells such as macrophages and/or DCs. ${ }^{41}$

The finding that the expression of CLM-1 is associated predominantly with eosinophils and not other myeloid cells is of quite interest, especially as, to date, the common knowledge was that CLM-1 or CD300f is mainly expressed by monocytes, DCs, and macrophages. ${ }^{23,28,42-44}$ The elevated baseline eosinophilia in $\mathrm{Clm} 1^{-/-}$mice in combination with the relatively eosinophil-associated expression pattern suggests that CLM-1 is constitutively active in eosinophils and that its ligand is readily accessible to these cells. Recent studies have indicated that CLM-1 (and additional CD300/CLM family members) can bind various ligands, including extracellular ceramide, phosphatidylcholine, sphingosyl, phosphatidylserine, and high-density and low-density lipoproteins. ${ }^{25,28,45,46}$ Directly related, we now show that CLM-1 binds apoptotic eosinophils and that phosphatidylserine is capable of suppressing eosinophil chemotaxis (at least in vitro), likely by interacting with CLM-1. These findings suggest that recruitment of eosinophils to sites of tissue damage and cell death ${ }^{47}$ is actively regulated by intrinsic negative feedback mechanisms such as CLM-1.
Furthermore, upon binding to ceramide, CLM-1 was capable of suppressing mast cell-induced activation, resulting in exacerbated mast cell-dependent allergic responses in $\mathrm{Clm} 1^{-1-}$ mice such as anaphylaxis, dermatitis, and airway inflammation. ${ }^{25}$ Certainly, intranasally OVA-challenged $\mathrm{Clm}^{-1-}$ mice displayed increased airway eosinophilia that was associated with increased Th2 cytokine production. ${ }^{25}$ Although increased eosinophilia in the latter study was attributed mainly to increased activation of mast cells, it is likely that increased eosinophil accumulation is regulated at least in part by direct negative signaling of CLM-1 in response to eotaxins. In support of this view, we demonstrate that blockade of CLM-1/ligand interactions in the effector stage of OVA-induced allergic inflammation induced a specific increase in eosinophil accumulation in the lungs. Although our in vivo studies cannot exclude the possible contribution of CLM-1 expression by additional myeloid cells, the finding that only eosinophil accumulation (but not neutrophil infiltration) was increased is consistent with our in vitro data that demonstrated a specific effect for CLM-1 in eotaxin-induced eosinophil (but not neutrophil) chemotaxis. Although our studies focused on eosinophils, it is possible that CLM-1 may regulate eotaxininduced responses in additional CCR3 ${ }^{+}$and eotaxin-responsive cells (e.g., basophils).

As many of the in vitro studies described herein were conducted using eosinophils that were obtained from longterm culture in the presence of IL-5, we cannot exclude a possible interaction between IL-5 and CLM- 1 that might affect eosinophil priming and subsequent migration. Nevertheless, this is unlikely as we observed increased eotaxin-induced chemotactic responses in vivo (in the absence of exogenous IL-5). Furthermore, inflammatory conditions promoting tissue eosinophilia are associated with increased IL- $5{ }^{48}$ Thus, our results may actually reflect how CLM-1 regulates eosinophils under disease conditions.

Finally, we demonstrate for the first time that CD300f is expressed by human eosinophils and that its expression is elevated in cells obtained from allergic rhinitis patients. This observation is important as it indicates that a factor in the inflammatory environment regulates the expression of CLM-1 that in turn can negatively regulate cellular responses in allergic settings, thereby providing a new inhibitory feedback mechanism in eosinophils.

In summary, our results establish a key role for CLM-1 in eosinophil homeostasis in health and disease. These data provide fundamental insights into negative regulation of eosinophils by inhibitory receptors (in general) and illustrate a model in which eosinophil tissue recruitment is constantly restrained by CLM-1 serving as a novel molecule restricting eotaxin-induced eosinophil homing signals.

\section{METHODS}

Mice. The generation of $C l m 1^{-1-}$ mice has been previously described. ${ }^{26} \mathrm{Clm}^{-/-}$mice (B6N.129S5-Cd300atm1Lex/Mmcd, ID 032182-UCD) were obtained from the Mutant Mouse Regional Resource Center (MMRC, University of California, Davis, CA; 
originally donated by Genentech) and backcrossed to C57BL/6 mice for 10 generations. WT C57BL/6 mice were originally obtained from Harlan Laboratories (Rehovot, Israel) and grown in-house. In all experiments, age-, weight-, and sex-matched mice were used and housed under specific pathogen-free conditions according to protocols approved by the Tel-Aviv University Institutional Animal Care Unit.

Mouse and human eosinophils. Mouse low-density BM-derived eosinophils were generated as previously described. ${ }^{49}$ Eosinophil purity was assessed by cytological analysis (Diff-Quick, Emmonya Biotech Ltd, Chelopech, Bulgaria) and CCR3/Sialic acid-binding immunoglobulin-like lectin-F (Siglec-F) positivity (flow cytometry) and were consistently $>90 \%$. For analysis of human eosinophils, blood samples were drawn from subjects treated at Meir Medical Center, Kfar Saba, Israel. Allergic patients were defined as subjects with a history of rhinitis and positive skin-prick test for at least one aeroallergen. The Institutional Review Board approved the study and all subjects signed an informed consent form. Before recruitment of the first subject, the protocol was registered at ClinicalTrials.gov (ID NCT01489293).

Generation of single-cell suspensions. Single-cell suspensions from the peritoneum, blood, BM, spleen, intestine, and adipose tissue (using the "walking out" technique) of WT, $C l m 1^{-1-}$, and $C l m 8^{-/-}$ mice were obtained as described previously. $3,37,50$

Flow cytometry. Single-cell suspensions of mouse cells were stained using the following antibodies: anti-CD45-PE-Cy7, anti-CD-45-APC, anti-CD11b-Pe, anti-MHC-II-PE-Cy7, anti-CD11c-Alexa Fluor 488, anti-Gr-1-FITC, anti-Gr-1-PE-Cy7, anti-Ly6C-PerCp-Cy5.5 (all obtained from eBioscience, San Diego, CA), anti-CD45-Brilliant Violet 570, rat IgG2a, Armenian hamster IgG (all obtained from Biolegend, San Diego, CA), anti-CCR3-FITC, anti-CLM-8 (all obtained from R\&D Systems, Minneapolis, MN), anti-Siglec-F-PE (BD Bioscience, San Jose, CA), anti-CLM-1 (provided by Dr Menno van Lookeren Campagne, Genentech South San Francisco, CA), antiArmenian-hamster-DyLight-649, and anti-rat-DyLight-649 (Jackson ImmunoResearch, West Grove, PA). For human peripheral blood stainings, the following anti-human antibodies were used: anti-CD16PE-Cy7, anti-CCR3-APC-Cy7 (all from Biolegend), anti-Siglec-8-PE (BD Biosciences, San Jose, CA), anti-CLM-1-Alexa Fluor 647, rat IgG1, and $\kappa$-Alexa Fluor 647 (all from eBioscience). Flow cytometry was performed using a 3-laser 10-color Gallios flow cytometer (Beckman Coulter, Brea, CA) and data were analyzed using Kaluza (Beckman Coulter) or FlowJo v10.3 (TreeStar, Ashland, OR) analysis software. All staining procedures were conducted following blocking with either $10 \%$ fetal calf serum or using an Fc-blocking reagent (eBioscience). Median fluorescent intensity (MFI) was obtained and $\triangle$ MFI calculated as previously described. ${ }^{37}$

Phosphoflow. Eotaxin-induced ERK-1/2 and p38 phosphorylation were assessed as previously described. ${ }^{38}$

Calcium influx. Eotaxin-induced calcium influx was assessed by flow cytometry using Fluo-4AM (4 mM, Invitrogen, Carlsbad, CA) according to the manufacturer's instructions.

Chemotaxis assay. In vitro chemotaxis assays were conducted as previously described. ${ }^{37}$ In several experiments, chemotaxis assays were performed in the presence of phosphatidylserine (Sigma, Rehovot, Israel). Alternatively, CLM-1/ligand interactions were blocked using an IgG1 CLM-1-Fc fusion protein $\left(5 \mu \mathrm{g} \mathrm{ml}^{-1}\right)$ or appropriate IgG1 control Fc fusion protein. ${ }^{26}$ In other experiments, single-cell suspensions from spleens were obtained and stained with anti-CD45, anti-Gr-1, and anti-CD11b for identification of neutrophils. Thereafter, the cells were washed and chemotaxis assays were performed. ${ }^{37}$

In vivo chemotaxis assay. Eotaxin- $1(1 \mu \mathrm{g}$ per $200 \mu \mathrm{l}$ saline) or saline were injected intraperitoneally into WT and $C l m 1^{-1-}$ mice. After $3 \mathrm{~h}$, the mice were killed, their peritoneal cavities washed, and cells were collected and counted. The percentage of eosinophils was determined by flow cytometry using anti-CD 45 , anti-Siglec-F, and anti-CD $11 \mathrm{~b}$ as described above.

Allergic eosinophilic airway inflammation. Allergic eosinophilic airway inflammation was induced in WT mice using OVA/Alum as described previously. ${ }^{51}$ CLM-1/ligand interactions were blocked by intranasal administration of CLM-1-Fc fusion protein or control IgG1 fusion protein $\left(20 \mu \mathrm{g} / \mathrm{ml}^{-1}, 1 \mathrm{~h}\right.$ before allergen challenge). Thereafter, bronchoalveolar lavage fluid was performed and accumulation of lung eosinophils assessed by cytological staining (Diff-Quick).

Enzyme-linked immunosorbent assay. Chemokines and cytokines were measured by enzyme-linked immunosorbent assay as described elsewhere. $^{51}$ The lower detection limits for CCL11, CCL24, and IL-5 were $7.8,15.6$, and $15.6 \mathrm{pg} \mathrm{ml}^{-1}$, respectively (Duo-Set, R\&D Systems). Peritoneal cavity chemokine levels were assessed after the peritoneal cavity was washed and the peritoneal fluid concentrated using centrifugal concentrators (3,000 molecular weight cutoff; Vivaspin, Gottingen, Germany).

Actin polymerization assay. Eosinophils were suspended in Hanks' balanced salt solution supplemented with $2 \%(\mathrm{w} / \mathrm{v})$ fetal calf serum and the cells were activated with eotaxin- $1\left(100 \mathrm{ng} \mathrm{ml}^{-1}, 37^{\circ} \mathrm{C}\right)$ for the indicated time points. The reaction was stopped (with $4 \%$ formaldehyde in phosphate-buffered saline) and cells were stained with fluorescein isothiocyanate (FITC)-conjugated phalloidin $\left(10 \mu \mathrm{g} \mathrm{ml}^{-1}\right.$; Sigma). Actin polymerization was measured by flow cytometry.

Statistical analysis. Data were analyzed by analysis of variance followed by Tukey's post hoc test or Student's $t$-test using GraphPad Prism 5 (San Diego, CA). Data are presented as mean \pm s.d., and values of $P<0.05$ were considered statistically significant.

SUPPLEMENTARY MATERIAL is linked to the online version of the paper at http://www.nature.com/mi

\section{ACKNOWLEDGEMENTS}

This study was supported by research funding to AYH (Morasha Program, Israel Science Foundation, grant 1084/10); to IM by the Joan and Jaime Constantiner Institute for Molecular Genetics; to AM by the FP7 MarieCurie Reintegration grant (grant 256311), the US-Israel Binational Science Foundation (grants 2009222 and 2011244), the Israel Science Foundation (grant 955/11), the Israel Cancer Research Foundation Research Career Development Award, the Dream Ideas Djerassi-Elias Institute of Oncology Research Fund, and internal Tel-Aviv University funds. We thank Drs Jamie and Nancy Lee (Mayo Clinic, Scottsdale, AZ) for the anti-MBP antibody and Dr Steffen Jung (Weizmann Institute, Rehovot, Israel) for providing critical reagents for this study. Itay Moshkovits performed this work in partial fulfillment of the requirements for a PhD degree at the Sackler Faculty of Medicine, Tel-Aviv University, Israel.

\section{AUTHOR CONTRIBUTIONS}

$\mathrm{IM}, \mathrm{MI}, \mathrm{DS}, \mathrm{DK}-\mathrm{A}, \mathrm{BB}$, and AM performed the experiments; IM, MI, AYH, and $A M$ designed the experiments and analyzed the data; MvLC analyzed the data and generated critical reagents for the study; IM and AM wrote the manuscript.

\section{DISCLOSURE}

Menno van Lookeren Campagne is an employee of Genentech; Ariel Munitz serves as a consultant for Immune Pharmaceuticals. The other authors declared no conflict of interest.

c 2014 Society for Mucosal Immunology

\section{REFERENCES}

1. Rosenberg, H.F., Dyer, K.D. \& Foster, P.S. Eosinophils: changing perspectives in health and disease. Nat. Rev. Immunol. 13, 9-22 (2013). 
2. Rothenberg, M.E. \& Hogan, S.P. The eosinophil. Annu. Rev. Immunol. 24 147-174 (2006).

3. Wu, D. et al. Eosinophils sustain adipose alternatively activated macrophages associated with glucose homeostasis. Science 332, 243-247 (2011).

4. Jacobsen, E.A., Taranova, A.G., Lee, N.A. \& Lee, J.J. Eosinophils: singularly destructive effector cells or purveyors of immunoregulation? J. Allergy Clin. Immunol. 119, 1313-1320 (2007).

5. Patel, V.P. et al. Molecular and functional characterization of two novel human C-C chemokines as inhibitors of two distinct classes of myeloid progenitors. J. Exp. Med. 185, 1163-1172 (1997).

6. Forssmann, U. et al. Eotaxin-2, a novel CC chemokine that is selective for the chemokine receptor CCR3, and acts like eotaxin on human eosinophil and basophil leukocytes. J. Exp. Med. 185, 2171-2176 (1997).

7. Shinkai, A. et al. A novel human CC chemokine, eotaxin-3, which is expressed in IL-4-stimulated vascular endothelial cells, exhibits potent activity toward eosinophils. J. Immunol. 163, 1602-1610 (1999).

8. Kitaura, M. et al. Molecular cloning of a novel human CC chemokine (Eotaxin-3) that is a functional ligand of CC chemokine receptor 3. J. Biol. Chem. 274, 27975-27980 (1999).

9. Jose, P.J. et al. Eotaxin: a potent eosinophil chemoattractant cytokine detected in a guinea pig model of allergic airways inflammation. J. Exp. Med. 179, 881-887 (1994)

10. Garcia-Zepeda, E.A., Rothenberg, M.E., Ownbey, R.T., Celestin, J., Leder, P. \& Luster, A.D. Human eotaxin is a specific chemoattractant for eosinophil cells and provides a new mechanism to explain tissue eosinophilia. Nat. Med. 2, 449-456 (1996).

11. Hogan, S.P. et al. A pathological function for eotaxin and eosinophils in eosinophilic gastrointestinal inflammation. Nat. Immunol. 2, 353-360 (2001).

12. Pope, S.M. et al. IL-13 induces eosinophil recruitment into the lung by an IL-5- and eotaxin-dependent mechanism. J. Allergy Clin. Immunol. 108, 594-601 (2001).

13. Pope, S.M. et al. Identification of a cooperative mechanism involving interleukin-13 and eotaxin-2 in experimental allergic lung inflammation. J. Biol. Chem. 280, 13952-13961 (2005).

14. Foster, P.S. et al. Elemental signals regulating eosinophil accumulation in the lung. Immunol. Rev. 179, 173-181 (2001).

15. Hogan, S.P., Mishra, A., Brandt, E.B., Foster, P.S. \& Rothenberg, M.E. A critical role for eotaxin in experimental oral antigen-induced eosinophilic gastrointestinal allergy. Proc. Natl. Acad. Sci. USA 97, 6681-6686 (2000).

16. Rosenberg, H.F., Phipps, S. \& Foster, P.S. Eosinophil trafficking in allergy and asthma. J. Allergy Clin. Immunol. 119, 1303-1310 (2007). (quiz 1311-1302).

17. Forbes, E. et al. Immunopathogenesis of experimental ulcerative colitis is mediated by eosinophil peroxidase. J. Immunol. 172, 5664-5675 (2004).

18. Bruijnzeel, P.L., Warringa, R.A., Kok, P.T., Hamelink, M.L., Kreukniet, H. \& Koenderman, L. Effects of nedocromil sodium on in vitro induced migration, activation, and mediator release from human granulocytes. J. Allergy Clin. Immunol. 92 (1 Pt 2), 159-164 (1993).

19. Huang, W.W., Garcia-Zepeda, E.A., Sauty, A., Oettgen, H.C., Rothenberg, M.E. \& Luster, A.D. Molecular and biological characterization of the murine leukotriene B4 receptor expressed on eosinophils. J. Exp. Med. 188, 1063-1074 (1998).

20. Busse, W.W. Leukotrienes and inflammation. Am. J. Respir. Crit. Care Med. 157 (6 Pt 2), S210-S213 (1998). discussion S247-218.

21. Munitz, A. Inhibitory receptors on myeloid cells: new targets for therapy? Pharmacol. Ther. 125, 128-137 (2010).

22. Lee, S.M., Suk, K. \& Lee, W.H. Synthetic peptides containing ITIM-like sequences of IREM-1 (CD300F) differentially regulate MyD88 and TRIFmediated TLR signalling through activation of SHP and/or PI3K. Clin. Exp. Immunol. 167, 438-446 (2012).

23. Lee, S.M., Kim, E.J., Suk, K. \& Lee, W.H. CD300F blocks both MyD88 and TRIF-mediated TLR signaling through activation of Src homology region 2 domain-containing phosphatase 1. J. Immunol. 186, 6296-6303 (2011).

24. Clark, G.J., Ju, X., Tate, C. \& Hart, D.N. The CD300 family of molecules are evolutionarily significant regulators of leukocyte functions. Trends Immunol. 30, 209-217 (2009).
25. Izawa, K. et al. The receptor LMIR3 negatively regulates mast cell activation and allergic responses by binding to extracellular ceramide. Immunity 37 , 827-839 (2012).

26. $\mathrm{Xi}, \mathrm{H}$. et al. Negative regulation of autoimmune demyelination by the inhibitory receptor CLM-1. J. Exp. Med. 207, 7-16 (2010).

27. Rothenberg, M.E. et al. Eotaxin triggers eosinophil-selective chemotaxis and calcium flux via a distinct receptor and induces pulmonary eosinophilia in the presence of interleukin 5 in mice. Mol. Med. 2, 334-348 (1996).

28. Choi, S.C. et al. Cutting edge: mouse CD300f (CMRF-35-like molecule-1) recognizes outer membrane-exposed phosphatidylserine and can promote phagocytosis. J. Immunol. 187, 3483-3487 (2011).

29. Fulkerson, P.C., Fischetti, C.A., McBride, M.L., Hassman, L.M., Hogan, S.P. \& Rothenberg, M.E. A central regulatory role for eosinophils and the eotaxin/CCR3 axis in chronic experimental allergic airway inflammation. Proc. Natl. Acad. Sci. USA 103, 16418-16423 (2006).

30. Munitz, A. \& Levi-Schaffer, F. Inhibitory receptors on eosinophils: a direct hit to a possible Achilles heel?. J. Allergy Clin. Immunol. 119, 1382-1387 (2007).

31. Hogan, S.P., Foster, P.S. \& Rothenberg, M.E. Experimental analysis of eosinophil-associated gastrointestinal diseases. Curr. Opin. Allergy Clin. Immunol. 2, 239-248 (2002).

32. Shik, D. \& Munitz, A. Regulation of allergic inflammatory responses by inhibitory receptors. Clin. Exp. Allergy 40, 700-709 (2010).

33. Zhu, D., Kepley, C.L., Zhang, K., Terada, T., Yamada, T. \& Saxon, A. A chimeric human-cat fusion protein blocks cat-induced allergy. Nat. Med. 11, 446-449 (2005).

34. Tam, S.W., Demissie, S., Thomas, D. \& Daeron, M. A bispecific antibody against human IgE and human FcgammaRIl that inhibits antigen-induced histamine release by human mast cells and basophils. Allergy 59, 772-780 (2004).

35. Verjan Garcia, N. et al. SIRPalpha/CD172a regulates eosinophil homeostasis. J. Immunol. 187, 2268-2277 (2011).

36. Zhang, H., Meng, F., Chu, C.L., Takai, T. \& Lowell, C.A. The Src family kinases Hck and Fgr negatively regulate neutrophil and dendritic cell chemokine signaling via PIR-B. Immunity 22, 235-246 (2005).

37. Munitz, A., McBride, M.L., Bernstein, J.S. \& Rothenberg, M.E. A dual activation and inhibition role for the paired immunoglobulin-like receptor $B$ in eosinophils. Blood 111, 5694-5703 (2008).

38. Munitz, A., Bachelet, I., Eliashar, R., Moretta, A., Moretta, L. \& Levi-Schaffer, F. The inhibitory receptor IRp60 (CD300a) suppresses the effects of IL-5, GM-CSF, and eotaxin on human peripheral blood eosinophils. Blood 107, 1996-2003 (2006).

39. Fulkerson, P.C. et al. Negative regulation of eosinophil recruitment to the lung by the chemokine monokine induced by IFN-gamma (Mig, CXCL9). Proc. Natl. Acad. Sci. USA 101, 1987-1992 (2004).

40. Martinez-Barriocanal, A., Comas-Casellas, E., Schwartz, S. Jr, Martin, M. \& Sayos, J. CD300 heterocomplexes, a new and family-restricted mechanism for myeloid cell signaling regulation. J. Biol. Chem. 285, 41781-41794 (2010)

41. Ahrens, R. et al. Intestinal macrophage/epithelial cell-derived CCL11/ eotaxin-1 mediates eosinophil recruitment and function in pediatric ulcerative colitis. J. Immunol. 181, 7390-7399 (2008).

42. Kim, E.J., Lee, S.M., Suk, K. \& Lee, W.H. CD300a and CD300f differentially regulate the MyD88 and TRIF-mediated TLR signalling pathways through activation of SHP-1 and/or SHP-2 in human monocytic cell lines. Immunology 135, 226-235 (2012).

43. Peluffo, $\mathrm{H}$. et al. Overexpression of the immunoreceptor CD300f has a neuroprotective role in a model of acute brain injury. Brain Pathol. 22, 318-328 (2012).

44. Chung, D.H., Humphrey, M.B., Nakamura, M.C., Ginzinger, D.G., Seaman, W.E. \& Daws, M.R. CMRF-35-like molecule-1, a novel mouse myeloid receptor, can inhibit osteoclast formation. J. Immunol 171, 6541-6548 (2003).

45. Borrego, F. The CD300 molecules: an emerging family of regulators of the immune system. Blood 121, 1951-1960 (2013).

46. Simhadri, V.R., Andersen, J.F., Calvo, E., Choi, S.C., Coligan, J.E. \& Borrego, F. Human CD300a binds to phosphatidylethanolamine and phosphatidylserine, and modulates the phagocytosis of dead cells. Blood 119, 2799-2809 (2012). 
47. Lee, J.J., Jacobsen, E.A., McGarry, M.P., Schleimer, R.P. \& Lee, N.A. Eosinophils in health and disease: the LIAR hypothesis. Clin. Exp. Allergy 40, 563-575 (2010).

48. Uhm, T.G., Kim, B.S. \& Chung, I.Y. Eosinophil development, regulation of eosinophil-specific genes, and role of eosinophils in the pathogenesis of asthma. Allergy Asthma Immunol. Res. 4, 68-79 (2012).

49. Dyer, K.D., Moser, J.M., Czapiga, M., Siegel, S.J., Percopo, C.M. \& Rosenberg, H.F. Functionally competent eosinophils differentiated ex vivo in high purity from normal mouse bone marrow. J. Immunol. 181, 4004-4009 (2008).

50. Zigmond, E. et al. Ly6C(hi) monocytes in the inflamed colon give rise to proinflammatory effector cells and migratory antigen-presenting cells. Immunity 37, 1076-1090 (2012).

51. Munitz, A., Bachelet, I. \& Levi-Schaffer, F. Reversal of airway inflammation and remodeling in asthma by a bispecific antibody fragment linking CCR3 to CD300a. J. Allergy Clin. Immunol. 118, 1082-1089 (2006). 